\title{
Anovulatory Infertility: A Prospective Study
}

\author{
Geetu Bhandoria, Samar Rudra
}

\begin{abstract}
Introduction: Anovulation is a common cause of infertility with poly cystic ovarian disease being the commonest.
\end{abstract}

Objective: To estimate the prevalence of various causes of anovulation in patients younger than 35 years of age attending infertility clinics and to ascertain the nature and extent of metabolic abnormalities and efficacy of therapy.

Materials and methods: Sixty cases of anovulatory infertility diagnosed by standard method were recruited for the study. Transvaginal sonography and hormonal profile like LH, FSH, Prolactin and Thyroid profile evaluated to establish the cause of anovulation. Menstrual history, Body Mass Index, Waist Hip ratio and presence of hirsutism, acanthosis nigricans were recorded. Metabolic parameters like lipid profile, OGTT and Glucose insulin ratio were also assessed. All parameters were reevaluated at 3 and 6 months of treatment and were statistically analyzed. Response to treatment in terms ovulation and pregnancy achieved was also analyzed.

Conclusion: Women with anovulatory infertility has shown good improvement to appropriate treatment.

Keywords: Infertility, Anovulation, Polycystic ovary syndrome, Clomiphen citrate.

How to cite this article: Bhandoria G, Rudra S. Anovulatory Infertility: A Prospective Study. Int J Infertility Fetal Med 2013; 4(1):18-23.

\section{Source of support: Nil}

Conflict of interest: None

\section{INTRODUCTION}

Infertility is rapidly becoming one of the leading causes of visits to gynecology clinic by couples in the reproductive age group. Anovulation is among the most common causes of infertility. ${ }^{1}$ WHO has devised a classification scheme for women who do not ovulate, dividing them into three major groups, i.e. Group I: Hypothalamic pituitary failure, who have low to low-normal gonadotropin secretion with menopausal estradiol concentrations, like Kallmann's syndrome, anorexia nervosa, etc; Group II: Hypothalamic pituitary dysfunction with estrogenic chronic anovulation, where individuals often exhibit elevated LH:FSH ratio, though gonadotropins are typically in the normal range, like PCOS; Group III: Hypergonadotropic hypogonadism where elevated levels of gonadotropins and hypoestrogenism is characteristic like premature ovarian failure. WHO Group II

Date of Received: 06-12-12

Date of Acceptance: 29-03-13

Date of Publication: January 2013 is by far the commonest group encountered in clinical practice. There are various methods available for assessing ovulation with advantages and pitfalls of each method. These methods are menstrual history, natural family planning methods, basal body temperature, serum progesterone concentration, urinary luteinizing hormone (LH) secretion monitoring, endometrial biopsy and transvaginal ultrasonography, though the only definitive proof of ovulation is pregnancy.

\section{OBJECTIVES}

1. To estimate the prevalence of various causes of anovulation in patients younger than 35 years of age attending infertility clinics.

2. To ascertain the nature and extent of metabolic abnormalities in such patients.

3. (i) To determine the efficacy of therapy in improving presenting complaints.

(ii) To determine the efficacy of therapy in improving metabolic abnormalities.

(iii) To determine the extent of ovulation and pregnancy achieved with treatment.

Study was conducted mainly on patients of poor to average socioeconomic status with a view to find various outcome in an average Indian population.

\section{MATERIALS AND METHODS}

This study was conducted at a tertiary care center from 01 Sep 2008 to 31 Mar 2010. The study population comprised of 60 women in the age group 21 to 34 years with anovulatory infertility. First 60 women identified with anovulatory infertility among a total of 167 attending infertility clinic were recruited in the study and each patient was studied for a period of 6 months. Patients were selected initially on the basis of menstrual history suggestive of anovulation (oligomenorrhea/amenorrhea), confirmed subsequently by transvaginal ultrasonographic follicular monitoring. Patients were also selected on the basis of TVUS when anovulation was found as cause of infertility even in presence of regular menses. A detailed menstrual, medical, surgical, drug and treatment history was taken. A thorough physical examination with particular reference to height, weight, body mass index (BMI), waist:Hip ratio, thyroid gland palpation, breast examination, body hair distribution, features of hyperinsulinemia (acanthosis nigricans) and gynecological examination was done. For anthropometric measurements, subjects were examined wearing light 
clothes. BMI was calculated as weight $(\mathrm{kg}) /$ height $\left(\mathrm{m}^{2}\right)$. Waist and hip circumferences were measured to the nearest centimeter with a tape measure while the subject was standing. The waist was measured at the smallest girth midway between the lowest rib margin and the iliac crest, and the hip circumference was measured at the level of the greater trochanter to calculate the waist/hip ratio. The clinical features of acanthosis nigricans were assessed. Hirsutism was classified according to the Ferriman-Gallwey score ( $>8$ taken as hirsutism). All patients were subjected to following investigation: Serum $\mathrm{LH}$, follicle stimulating hormone (on day 2 of cycle) (Abbott Labs kit by microenzyme immunoassay), serum testosterone (Abbott Labs kit by microenzyme immunoassay), thyroid function tests (Abbott Labs kit by microenzyme immunoassay), prolactin levels (Abbott Labs kit by Microenzyme immunoassay), lipid profile, fasting glucose, fasting serum insulin (Biomedics Labs kit by microenzyme immunoassay), 75 gm 2-hour glucose tolerance test and transvaginal ultrasound (GE Healthcare Ultrasound LOGIQ P5/LOGIQ A5, 6.5 MHz E8C TVUS Probe). Patients were then diagnosed as PCOS/thyroid disorders/hyperprolactinemia/ combination of these disorders on basis of standard diagnostic criteria. Patients were treated on basis of diagnosis:

1. PCOS

a. Weight reduction

b. Insulin sensitizer - tab Metformin $500 \mathrm{mg}$ TDS.

2. Hypothyroidism: Tab Eltroxin starting at 25 to $50 \mu \mathrm{g} \mathrm{OD}$ escalated as per requirement.

3. Hyperthyroidism: Tab carbimazole $10 \mathrm{mg}$ TDS titrated as per thyroid status.

4. Hyperprolactinemia: Tab bromocriptine $2.5 \mathrm{mg}$ OD-BD. After achieving ovulation, controlled ovarian hyper stimulation $(\mathrm{COH})$ was achieved with clomiphene citrate/ purified $\mathrm{FSH}$ and later ovulation trigger with injection human chorionic gonadotropin ( $\mathrm{hCG}$ ) was given. $\mathrm{COH}$ was done even if ovulation was not achieved after 3 cycles of treatment, as primary complaint of patients was infertility. This was followed by timed intercourse/intrauterine insemination (IUI). Change in menstrual history pattern in patients with abnormal menses, anthropometric parameters like waist:hip ratio; biochemical parameters like fasting glucose:insulin ratio and serum testosterone were measured at 3 and 6 months after treatment. Hormonal variables, like serum LH, FSH, LH:FSH ratio, prolactin, thyroid hormone levels, were measured at 3 and 6 months following treatment. The results thus obtained were analyzed statitistically using students' t-test.

\section{RESULTS}

A total of 60 patients who met the selection criteria were recruited in the study, out of the first 167 infertile patients attending the outpatient clinic. On further evaluation, $38(63.3 \%)$ patients were diagnosed to have features of polycystic ovary syndrome, eight (13.3\%) had hyperprolactinemia, six (10\%) had hypothyroidism, two (3.3\%) had hyperthyroidism and six (10\%) had combination of above disorders. Our study found PCOS as dominating cause of anovulation among the infertile population with as high as $63.3 \%$ of cases.

Table 1 shows distribution of patients with menstrual irregularities and their response to treatment. Twenty-eight patients out of 39 with menstrual irregularities showed improvement following treatment. Around $71.4 \%$ of patients showed improved menses 6 months following treatment with weight reduction and metformin in PCO group and similar improvement found with appropriate treatment in other groups.

The various metabolic derangements found in PCOS patients are highlighted in Table 2. Current study showed a prevalence of $13.1 \%$ obesity (BMI $\geq 30$ ) and $34.2 \%$ overweight individuals (overall 47.3\%). Mean waist:hip ratio found in this study was 0.76 , which is on the higher side of the normal levels. Abnormal lipid profile was found in $15.8 \%$ of PCO patients, without any improvement over 6 months of treatment. Our study found mean G:I ratio to be 2.957 indicating insulin resistance. G:I ratio as well as those showing abnormal OGTT did not show any appreciable improvement in the values after treatment. $13(34 \%)$ patients showed free testosterone levels $>60 \mathrm{ng} / \mathrm{dl}$ indicating hyperandrogenemia. There was a significant fall 3 and 6 months post-treatment $(60.5 \mathrm{ng} / \mathrm{dl}$ at 3 months and $54.60 \mathrm{ng} / \mathrm{dl}$ at 6 months following treatment vs $76.9 \mathrm{ng} / \mathrm{dl}$ before treatment). Table 3 shows that mean LH level was 12.092 IU/L and FSH level was 3.36 IU/1 in this study. Mean LH: FSH ratio in this study was 3.799 . Values $\geq 3.0$ is considered to be a feature of PCOS. ${ }^{10}$ After treatment, LH levels showed a significant fall (10.951 vs 12.103 at 3 months, p-value 0.00 and 10.86 vs 12.103 at 6 months, p-value 0.010), the levels were however still high. There was a modest fall in LH:FSH ratio 3 and 6 months posttreatment which reached statistical significance (3.3 vs 3.7, p-value 0.012 at 3 months and 3.1 vs 3.7, p-value 0.010 at 6 months).

There were total eight patients presenting with hyperprolactinemia as cause of anovulatory infertility and two patients who had hyperprolactinemia with PCOS and one patient had coexistent hypothyroidism in current study. The basal prolactin levels ranged from 33.0 to $121.8 \mathrm{ng} / \mathrm{ml}$ (mean $57.08 \mathrm{ng} / \mathrm{ml}$ ). Following bromocriptine treatment $(2.5$ mg BD daily), mean prolactin levels came down to $22.42 \mathrm{ng} / \mathrm{ml}$ at 3 months and $15.93 \mathrm{ng} / \mathrm{ml}$ at 6 months (Table 3). One patient with basal prolactin level $121.8 \mathrm{ng} / \mathrm{ml}$ 
was subjected to MRI brain to rule out macroadenoma; however, the study showed no adenoma. After continued treatment with bromocriptine, the prolactin levels came down to within normal range in all the patients.

There were total eight patients presenting only with thyroid abnormalities as cause of anovulatory infertility and six patients had hypothyroidism with either PCOS or hyperprolactinemia in the current study. Out of total 14 patients with thyroid disorders, only two patients had hyperthyroidism, while rest of the patients presented with hypothyroidism. They responded well to appropriate treatment with improvement in symptoms as well as hormonal profile. Their hormonal profile values before and after treatment is shown in Table 3.
Table 4 shows ovulation study in patients studied. $78.9 \%$ of PCO patients and all other achieved ovulation during the study period. Table 5 shows the pregnancies achieved during 6 months follow-up period. A total of 19 pregnancies were achieved during this study. Ten out of 38 PCO patients became pregnant during the study. There were two spontaneous pregnancies with bromocriptine alone and two pregnancies with bromocriptine and ovulation induction with clomiphene followed by intrauterine insemination making total pregnancy rate achieved 50\% among hyperprolactinemia patients. There was one spontaneous pregnancy during treatment with thyroxine only and two pregnancies with thyroxine and ovulation induction with clomiphene followed by intrauterine insemination in

Table 1: Menstrual patterns among various groups of patients and response to treatment in such patterns

\begin{tabular}{|c|c|c|c|c|c|}
\hline S. no. & Pattern & PCOS; $n(\%)$ & Hyperprolactinemia; n (\%) & Thyroid disorders; $n$ (\%) & Combined; $n$ (\%) \\
\hline 1. & Irregular & $28(73.7)$ & $3(37.5)$ & $5(62.5)$ & $3(50.0)$ \\
\hline 2. & Regular & $10(26.3)$ & $5(62.5)$ & $3(37.5)$ & $3(50.0)$ \\
\hline 3. & Total & 38 & 8 & 8 & 6 \\
\hline 4. & Improved & $20(71.4)$ & $2(66.7)$ & $4(80)$ & $2(66.7)$ \\
\hline 5. & Not improved & $8(28.6)$ & $1(33.3)$ & $1(20.0)$ & 1 (33.3) \\
\hline
\end{tabular}

Table 2: Various metabolic disorders in patients of polycystic ovaries alone or in combination with other anovulatory disorders

\begin{tabular}{|c|c|c|c|c|c|c|c|c|c|c|}
\hline \multirow[t]{2}{*}{ Group } & \multicolumn{2}{|c|}{ Waist: hip ratio } & \multicolumn{2}{|c|}{ Hirsutism } & \multicolumn{2}{|c|}{ Acanthosis } & \multicolumn{2}{|c|}{ Lipid profile } & \multicolumn{2}{|c|}{ GTT } \\
\hline & Increased & Not increased & Present & Absent & Present & Absent & Normal & Abnormal & Normal & Abnormal \\
\hline $\begin{array}{l}\text { PCOS; } \\
\text { n (\%) }\end{array}$ & 7 (18.4) & 31 (81.6) & $23(60.5)$ & 15 (39.5) & 12 (31.6) & $26(68.4)$ & 32 (84.2) & $6(15.8)$ & $34(89.5)$ & $4(10.5)$ \\
\hline $\begin{array}{l}\text { Combined; } \\
\text { n (\%) }\end{array}$ & $1(16.7)$ & 5 (83.3) & $0(0)$ & $6(100)$ & $2(33.3)$ & $4(66.7)$ & $6(100)$ & $0(0)$ & 5 (83.3) & $1(16.7)$ \\
\hline
\end{tabular}

Table 3: Comparative statistical analysis of various hormonal markers in this study

\begin{tabular}{|c|c|c|c|c|c|}
\hline Variable & Baseline & 3 months & 6 months & $\begin{array}{l}p \text {-value (base vs } \\
3 \text { months) }\end{array}$ & $\begin{array}{l}p \text {-value (base vs } \\
6 \text { months) }\end{array}$ \\
\hline LH (IU/I) & $12.09(38) \pm 1.52$ & $10.95(38) \pm 1.39$ & $10.86(38) \pm 1.26$ & 0.00 & 0.010 \\
\hline FSH (IU/I) & $3.36(38) \pm 0.78$ & $3.26(38) \pm 0.78$ & $3.10(38) \pm 0.70$ & 0.628 & 0.620 \\
\hline LH:FSH & $3.79(38) \pm 1.02$ & $3.30(38) \pm 0.75$ & $3.11(38) \pm 0.46$ & 0.012 & 0.010 \\
\hline G:I ratio & $2.95(38) \pm 0.68$ & $2.97(38) \pm 0.58$ & $2.86(38) \pm 0.85$ & 0.853 & 0.839 \\
\hline Prolactin (mU/l) & $57.08(12) \pm 19.88$ & $22.42(12) \pm 5.85$ & $15.93(12) \pm 2.17$ & 0.000 & 0.001 \\
\hline TSH $(\mathrm{mU} / \mathrm{l})$ & $24.54(8) \pm 14.92$ & $12.57(8) \pm 5.11$ & $5.98(8) \pm 2.07$ & 0.0021 & 0.010 \\
\hline
\end{tabular}

\begin{tabular}{llclll}
\multicolumn{8}{c}{ Table 4: Ovulation achieved after treatment in various subset of patients } \\
\hline S. no. & & PCOS; $n(\%)$ & Hyperprolactinemia; $n(\%)$ & Thyroid disorders; $n(\%)$ & Combined; $n(\%)$ \\
\hline 1. & Achieved & $30(78.9)$ & $8(100)$ & $8(100)$ & $6(100)$ \\
2. & Not achieved & $8(21.1)$ & 0 & 0 & 0 \\
\hline
\end{tabular}

\begin{tabular}{llllll}
\multicolumn{8}{c}{ Table 5: The number of pregnancies achieved in various subgroups } \\
\hline S. no. & Pregnancy & PCOS; $n(\%)$ & Hyperprolactinemia; $n(\%)$ & Thyroid disorders; $n$ (\%) & Combined; $n(\%)$ \\
\hline 1. & Achieved & $10(26.3)$ & $4(50.0)$ & $3(37.5)$ & $2(33.3)$ \\
2. & Not achieved & $28(73.7)$ & $4(50.0)$ & $5(62.5)$ & $4(66.7)$ \\
3. & Total & 38 & 8 & 8 & 6 \\
\hline
\end{tabular}


hypothyroid patients. No pregnancy could be achieved in either of the two hyperthyroid patients.

\section{DISCUSSION}

Anovulation is a common cause underlying infertility and is a common feature of various disorders like PCOS, thyroid disorders, etc. Overall 60 to $85 \%$ of such patients demonstrate overt menstrual dysfunction, primarily oligoamenorrhea, the remainder present with apparent eumenorrhea; although about 5\% may demonstrate polymenorrhea. With treatment the length of menstrual cycle decreased from 45 to 120 days to 35 to 40 days, a $40 \%$ decrease in our study. In the study by Kolodziejczyk et al, the average length of cycle declined by $36 \%{ }^{2}$

True virilization is rare in PCOS, $70 \%$ of them complain of cosmetically disturbing hirsutism. Adams et $\mathrm{al}^{3}$ showed hirsutism in $55 \%$ of his patients. Our study showed hirsutism in $39.5 \%$ of PCOS patients. No change in hirsutism was seen after 6 months of treatment. $60 \mathrm{ng} / \mathrm{dl}$ of free serum testosterone was taken as the cutoff for hyperandrogenemia in this study. The free testosterone level ranged from 23.06 to $112.89 \mathrm{ng} / \mathrm{dl}, 13$ (34\%) patients showed levels $>60 \mathrm{ng} / \mathrm{dl}$ indicating hyperandrogenemia. There was significant fall 3 and 6 months post-treatment $(60.5 \mathrm{ng} / \mathrm{dl}$ at 3 months and $54.60 \mathrm{ng} / \mathrm{dl}$ at 6 months following treatment vs $76.9 \mathrm{ng} / \mathrm{dl}$ before treatment). Similar results were found by Hahn et al. ${ }^{4}$ The prevalence of obesity in PCOS women has been reported to be a little more than $50 \%$ of cases. ${ }^{5}$ Our study found $47.3 \%$ overweight patients among PCOS subgroup. Waist:hip ratio $>0.85$ is generally considered a marker of insulin resistance. ${ }^{5}$ Eight patients only had raised waist:hip ratio in our study. Waist:hip ratio was significantly higher in PCOS women than in control women in Legro et al study. ${ }^{6}$ A ratio of fasting glucose $(\mathrm{G})$ to fasting insulin (I) has been qualified as a simple and useful predictor of insulin resistance in women with PCOS. A fasting G:I ratio (cutoff value $<4.5$ ) provided the best combination of sensitivity (95\%) and specificity (84\%) as well as the best positive predictive value ( $87 \%$ ) and negative predictive value $(94 \%)$ as a screening test for predicting insulin resistance in PCOS. The glucose-stimulated parameters obtained from the OGTT, although sensitive, displayed less specificity than fasting insulin and/or the fasting G:I ratio. ${ }^{6}$ Insulin resistance and the resulting hyperinsulinemia contribute to the reproductive abnormalities in $\mathrm{PCO}$ women. There was no significant rise in fasting G:I ratio after 6 months of treatment in our study. Trolle et al too found no significant improvement in their study. ${ }^{7}$ Present study did not find any improvement in insulin sensitivity possibly because of less number of obese individuals affecting overall results. All women with PCOS are at increased risk of developing impaired glucose tolerance and overt type 2 diabetes mellitus due to underlying insulin resistance. In Legro et al study IGT was found in 31\% of women with PCOS and diabetes in $7.5 \%$. In nonobese, these figures were 10.3 and $1.5 \% .^{6}$ Present study found abnormal OGTT in four PCOS patients and one with combination disorders, which showed no improvement over 6 months treatment, probably due to short period of observation. Pasquali et al ${ }^{8}$ demonstrated improvement in carbohydrate metabolism in his study over a period of 2 years, in comparison to our studies of 6 months duration. A spectrum of abnormal lipid and lipoprotein profiles may be found in patients with PCOS; characteristically patients have raised cholesterol, triglycerides, LDL and decreased HDL and Apo A1 levels. These findings, however, are highly variable and depend on the obesity status, diet and ethnicity of the population studied. Yilmaz et al found no positive impact on serum lipids in their 6 months study of PCOS patients, ${ }^{9}$ similar to our study.

LH and FSH levels were measured and LH levels (mean LH level: $12.092 \mathrm{IU} / \mathrm{l})$ were found to be raised in all PCOS patients in this study. Normal levels in early follicular phase $6.5 \mathrm{IU} / 1$, mid-follicular phase $5.0 \mathrm{IU} / 1$, late follicular phase $7.2 \mathrm{IU} / 1 .{ }^{10}$ Nestler et al showed a significant reduction in basal levels of LH with metformin therapy (from $8.5 \mathrm{mIU} / 1$ to $2.8 \mathrm{mIU} / \mathrm{ml}$ ) in both obese as well as lean PCOS patients. ${ }^{11}$ Our study did not show such a fall in LH levels probably due to less number of individuals achieving weight reduction over 6 months affecting both G:I ratio as well as LH levels.

Follicular maturation (follicles $>15 \mathrm{~mm}$ ) was assessed during the study period. $78.9 \%$ of PCOS patients, all patients of thyroid disorders, hyperprolactinemia and combination disorders achieved ovulation following treatment. Follicular maturation was seen by TVUS starting from day 10 of the menstrual cycle. Pirwani et al showed improvement in ovulation ranging from 40 to $90 \%{ }^{12}$ There were two (5.5\%) spontaneous pregnancies following metformin treatment in PCOS group. Velazquez et al described $11 \%$ spontaneous pregnancies in his study. ${ }^{13}$ Rest 36 patients underwent ovulation induction with clomiphene citrate followed by timed intercourse (TI) or intrauterine insemination (IUI). There were eight pregnancies (22.2\%) following ovulation induction with TI/IUI, making an overall pregnancy rate $26.3 \%$. Results could have been better if the study was for a longer duration. George et al achieved overall pregnancy rate of $16.7 \%$ in 27 patients who were given metformin $1,500 \mathrm{mg} /$ day in divided doses for 6 months. ${ }^{14}$

Hyperprolactinemia commonly occurs in the absence of galactorrhea $(66 \%)$, which may result from inadequate estrogenic or progestational priming of breast. ${ }^{1} 63.6 \%$ 
(total 7) of the patients in our study had galactorrhea at the time of presentation, while $36.4 \%$ (4 patients) did not have galactorrhea and pregnancy achieved in this group is $50 \%$. Pepperell et al had found $16(80 \%)$ out of 20 patients of hyperprolactinemia to be having clinical galactorrhea. Basal prolactin levels in their study ranged from 19.0 to $93.2 \mathrm{ng} / \mathrm{ml}$ and ovulation achieved in $17(85 \%)$ of the 20 patients studied and pregnancy in $14(70 \%){ }^{15}$

Krass et al found menstrual irregularities in 23 to $25 \%$ of patients with thyroid disorders in their study, oligomenorrhea was found to be commonest manifestation. ${ }^{16}$ In our study, six (75\%) of these patients reported improved cycles after 6 months of treatment. There was a significant drop in TSH levels with thyroxine associated with clinical improvement in the form of ovulation. Though the TSH levels showed a significant fall following treatment, the mean levels were still high (5.986 at 6 months); this was probably due to a few patients with initial very high levels affecting overall figures. Our pregnancy rate achieved in this group is $21 \%$. Oravec et al reported pregnancy rates following thyroxine replacement therapy in infertile women with ovulatory dysfunction and hypothyroidism up to $64 \% .{ }^{17}$ Becker et al showed overall and spontaneous pregnancy rates to be highest among women with normal TSH levels following treatment. ${ }^{18}$

\section{CONCLUSION}

Anovulation is a common cause of female infertility. Polycystic ovary syndrome by far the commonest underlying factor. It is associated with a number of metabolic and endocrinological abnormalities principally hyperinsulinemia and hyperandrogenism. Metformin improves menstrual cyclicity, follicular maturation and ovulation rates in PCOS patients besides improving the metabolic parameters which is of great significance in preventing the long-term consequences. Dopamine agonists like bromocriptine can correct hormonal and ovulatory dysfunction associated with hyperprolactinemia. Appropriate treatment of thyroid disorders in form of thyroxine replacement in hypothyroid and antithyroid drugs in hyperthyroid individuals can improve menstrual and ovulatory disturbances in such patients.

Success rate of treatment is much higher in patients with anovulatory infertility in comparison to other causes of female infertility. Management of such cases can easily be handled by gynecologists at peripheral centers with basic infrastructure.

Findings of this study in respect of various anthropometric, metabolic and endocrinological parameters as well as their response to treatment in this subset of patients, require further evaluation by a larger and long-term study in this socioeconomic and ethnic group of population.

\section{REFERENCES}

1. Speroff L, Fritz MA. Clinical gynecological endocrinology and infertility (7th ed). Lippincott Williams and Wilkins 2010.

2. Kolodziejczyk B. Metformin decreases hyperandrogenism and hyperinsulinemia in women with PCOS. Fertl Stril 2000;73: 6-12.

3. Adams J, Polson DW, Franks S. Prevalence of polycystic ovaries in women with anovulation and idiopathic hirsutism. BMJ 1986; 293:355-59.

4. Hahn S, Benson S, Elsenbruch S, Pleger K, Tan S, Mann K, et al. Metformin treatment of polycystic ovary syndrome improves health-related quality-of-life, emotional distress and sexuality. Human Reprod 2006;21(7):1925-34.

5. Korhonen S, Hippeläinen M, Niskanen L, Vanhala M, Saarikoski S. Relationship of the metabolic syndrome and obesity to polycystic ovary syndrome: A controlled, populationbased study. Am J Obstet Gynecol 2001;184:289-96.

6. Legro RS, Kunselman AR, Dodson VC, et al. Prevalence and predictions of the risk for type 2 diabetes mellitus and impaired glucose tolerance in polycystic ovary syndrome: A prospective, controlled study in 254 affected women. J Clin Endocrinol Metab 1999;84:165-74.

7. Trolle B, Flyvbjerg A, Kesmodel U, Lauszus FF. Efficacy of metformin in obese and non-obese women with polycystic ovary syndrome: A randomized, double-blinded, placebo-controlled cross-over trial. Hum Reprod 2007;22(11):2967-73.

8. Pasquali R, Gambineri A, Biscotti D, et al. Effect of long-term treatment with metformin added to hypocaloric diet on body composition, fat distribution, and androgen and insulin levels in abdominally obese women with and without the polycystic ovary syndrome. J Clin Endocrinol Metab 2000;85:2767-74.

9. Yilmaz M, Bukan N, Ayvaz G, Karakoç A, Törüner F, Çakir N, Arslan M. The effects of rosiglitazone and metformin on oxidative stress and homocysteine levels in lean patients with polycystic ovary syndrome. Hum Reprod 2005;20(12):3333-40.

10. Rajaniemi HJ, Ronnberg L, Kauppila A, Ylostalo P, Vihko R. Luteinizing hormone receptors in ovarian follicles of patients with polycystic ovarian disease. J Clin Endocrinol Metab 1980;51:1054-57.

11. Nestler JE, Jakubowicz DJ. Lean women with polycystic ovary syndrome respond to insulin reduction with decreases in ovarian P450c17_activity and serum androgens. J Clin Endocrinol Metab 1997;82:4075-79.

12. Pirwany IR, Yates RW, Cameron IT, Fleming R. Effects of the insulin sensitizing drug metformin on ovarian function, follicular growth and ovulation rate in obese women with oligomenorrhoea. Hum Reprod 1999;14:2963-68.

13. Velazquez EM, Mendoza S, Hamer T, et al. Metformin therapy in polycystic ovary syndrome reduces hyperinsulinemia, insulin resistance, hyperandrogenemia, and systolic blood pressure, while facilitating normal menses and pregnancy. Metabolism 1994;43:647-54.

14. George SS, George K, Irwin C, Job V, Selvakumar R, Jeyaseelan V, Seshadri MS. Sequential treatment of metformin and clomiphene citrate in clomiphene-resistant women with polycystic ovary syndrome: A randomized, controlled trial. Hum Reprod 2003;18(2):299-304. 
15. Pepperell RJ, Evans JH, Grown JB, Smith MA, Healy D, Burger HG. Serum prolactin levels and the value of bromocriptine in the treatment of anovulatory infertility. BJOG 1977;84:58-66.

16. Krassas GE, et al. Thyroid disease and female reproduction. Fertil Steril 2000;74(6):1063-70.

17. Oravec S, Hlavacka S. Disorders of thyroid function and fertility disorders. Ceska Gynekol 2000;65(1):53-57.

18. Gerhard I, Becker T, Eggert-Kruse W, Klinga K, Runnebaum B. Thyroid and ovarian function in infertile women. Hum Reprod 1991;6(3):338-45.

\section{ABOUT THE AUTHORS}

\section{Geetu Bhandoria}

Major, Department of Obstetrics and Gynecology, Armed Forces Medical College, Pune, Maharashtra, India

\section{Samar Rudra (Corresponding Author)}

Professor, Department of Obstetrics and Gynecology, MM Institute of Medical Science and Research, Rohtak, Haryana, India, e-mail: rudra_samar@yahoo.co.in 